\title{
SIMULATING SATELLITE DOWNLINK DATA LOSS AND RECOVERY DUE TO RAIN ATTENUATION
}

\author{
Douglas Shannon \\ GreenDart Incorporated \\ 302 W. 5th Street, Suite 309 \\ San Pedro, CA 90731-2750 USA
}

\author{
Richard Marymee \\ GreenDart Incorporated \\ 302 W. 5th Street, Suite 309 \\ San Pedro, CA 90731-2750 USA
}

\begin{abstract}
This paper describes a simulation tool and trade study that models polar orbiting weather satellites, stored sensor data, downlinks to global receptors, and sensor data retrieval with sufficient fidelity to conduct design trades in autonomous satellite downlinks. This paper employs a stochastic rain model based on empirical rain data and a rain fade model for simulated data loss. Models have been implemented using the simulation tool ExtendSim with orbital data imported from Satellite Tool Kit (STK). The simulated satellite contacts and receptor environment are realistically modeled for data retrieval and lost data recovery.
\end{abstract}

\section{INTRODUCTION}

There are two alternatives for retrieving satellite data: autonomous downlink and commanded downlink, with complex trades for cost, complexity, data latency, and data reliability associated with data loss/recovery due to signal attenuation caused by rain. The satellite contact with a commanded downlink assures that the satellite is in range, in Line of Sight (LoS), and has the signal strength sufficient to downlink data with minimal risk of data loss, but it has the added cost and complexity of an uplink antenna, a command \& control center, and possibly an on-site operator for the commanding. The autonomous downlink is considerably simpler and cheaper to build with a contact schedule to track and receive satellite downlinks at predicted pointing and times, but it is ignorant of the dynamic weather conditions that could lose data at the receptor.

This paper examines the simulated loss and recovery of satellite data in the autonomous downlink due to rain at global satellite ground receptors using a stochastic rain model. Data signal loss due to rain is exponentially greater at lower antenna elevation angles as the satellite ascends at the start and descends at the end of contact. Data loss is a function of the elevation angle (i.e., the LoS distance through the atmosphere) and the rainfall rate. This paper uses a simplified function for the rain attenuation for a range, elevation angle and rain density to generate a probability of data loss. The paper includes a description of a satellite system simulation that models the sensor data stored between the contacts and data downlinks at the global receptors. The paper discusses alternative architectures for a notional ground receptor at Guam by comparing the data latency and data loss from rain attenuation.

Modeling data latency from satellite sensor collection to the generation of weather products as discussed in this paper was a small facet of a prior simulation effort (Shannon 2008) that supported the NASA/NOAA for the Suomi National Polar-orbiting Partnership (NPP) program. The tool discussed in this paper adds the impact of data loss and recovery on data latency. It was developed for GreenDart Inc. to support future Air Force Weather Agency (AFWA) programs like the Weather Satellite Follow-On Activities (WSFA) Risk Reduction Technology Project. 


\section{Shannon and Marymee}

\section{MODELING RAIN RATE AND RAIN ATTENUATION}

\subsection{Rainfall and Rain Rate}

Empirical rain data for Andersen AFB Guam (freemeteo.com, Lander 2003) was analyzed for a simple rain probability model. Figure 1 is a plot of ten years (2003-2012) historical weather rain measurements for Guam; missing data is shown in red. The simulation described in this paper uses Guam's summer rainfall for June through August for the data loss/recovery models and design analyses. The rain density model is driven by three rain probability profiles: consecutive dry days, consecutive rain days, and rain levels on rainy days. Profiles are calculated for each season; the summer in Andersen AFB Guam is highlighted below.

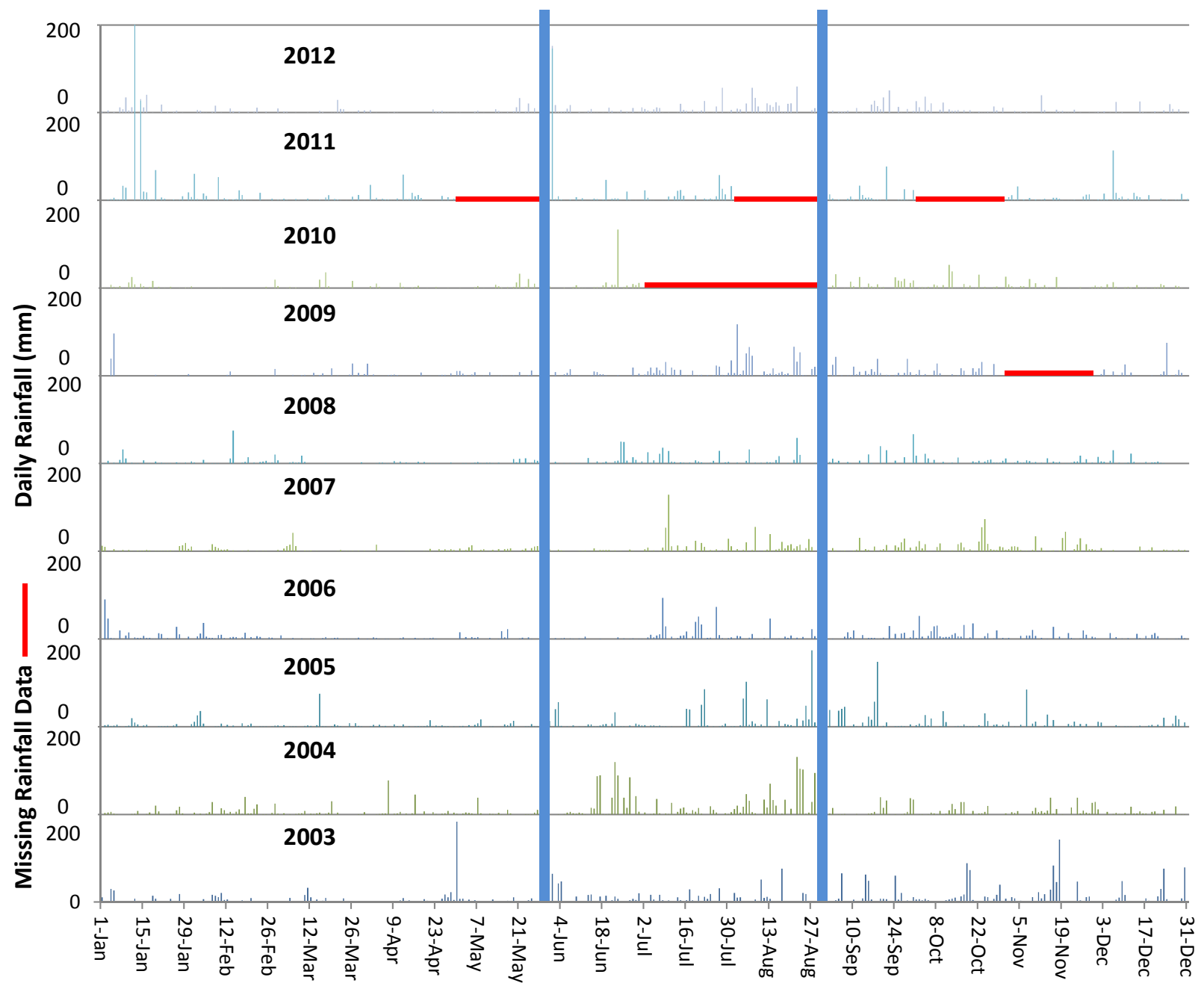

Figure 1: Andersen AFB Guam: Ten Years Measured Daily Rain Levels (mm)

Figure 2 shows the probability (i.e., pmf) of the sequential number of dry days and sequential wet days for summer in the years 2003-2012 with the meaningful rain threshold set as a daily total of 0.25 inches $(6.35 \mathrm{~mm})$ or greater. In the model, given a day is rainy, this profile gives the probability that the second (or third, etc.) day will be rainy. Later we discuss how the simulated rain daily total with the stochastic 


\section{Shannon and Marymee}

rain rate calculates rain periods, rain rates and resulting data loss at or below derived antenna elevation angles. Mapping the daily rain volume to a number of 2 or 3 hour periods with rain attenuation in the day is a sufficient level of fidelity for the data loss model.

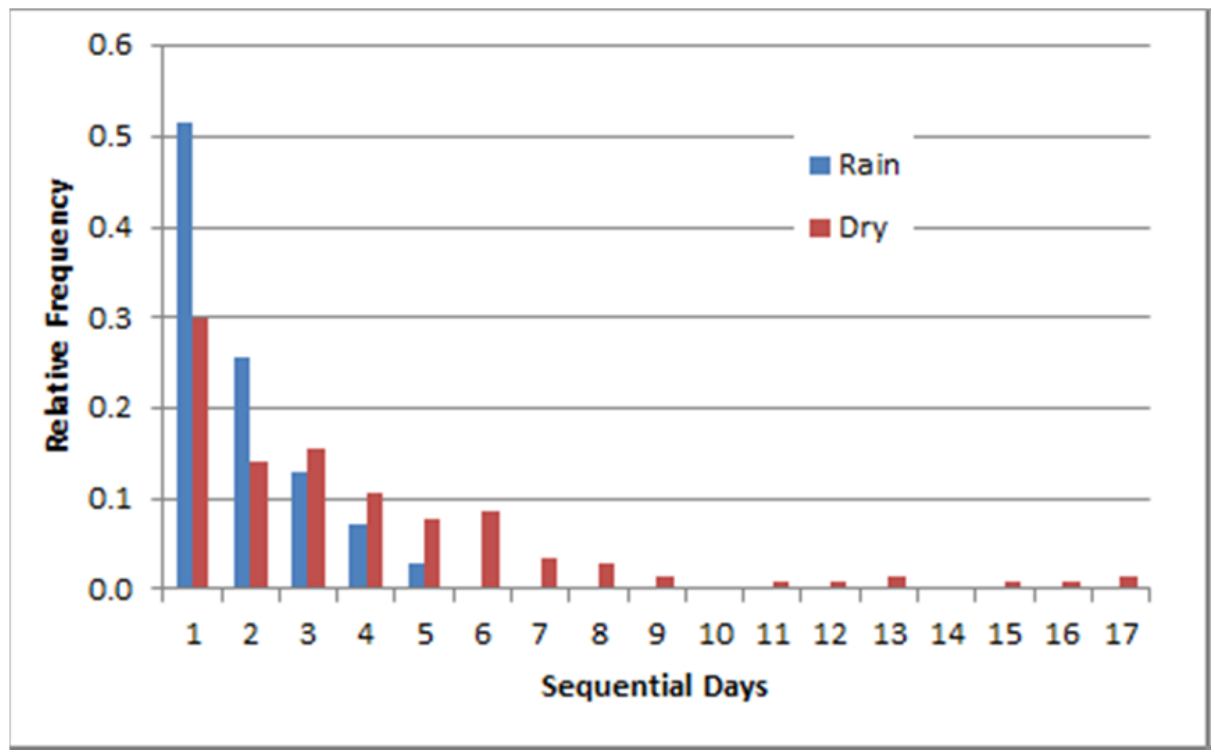

Figure 2: Relative Frequency of Sequential Dry or Wet Days

Given the day has meaningful rain, i.e., more than $6.35 \mathrm{~mm}$, Figure 3 is the profile of daily total rain volume in $\mathrm{mm} /$ day. The simulation in Section 3 uses the frequency of wet days above and the daily rain volume in Figure 3 to model rain rate and data loss. As seen in ten years of rain data in Figure 1, there are substantial rain levels that translate into long periods of high rain rates and great data loss.

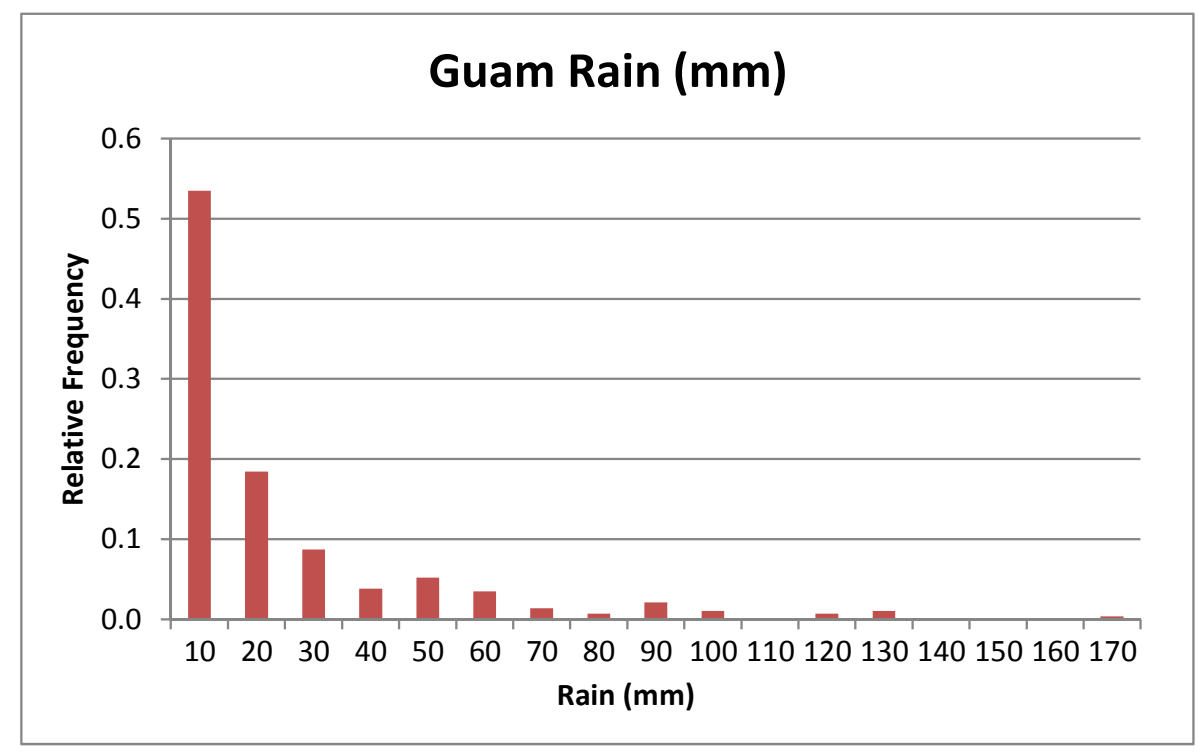

Figure 3: Probability Distribution for Daily Rain Total (in mm) 


\section{Shannon and Marymee}

Figure 4 compares modeled rain activity to the three year actuals from Figure 1 and shows a realistic frequency and daily rate volume $(\mathrm{mm})$.

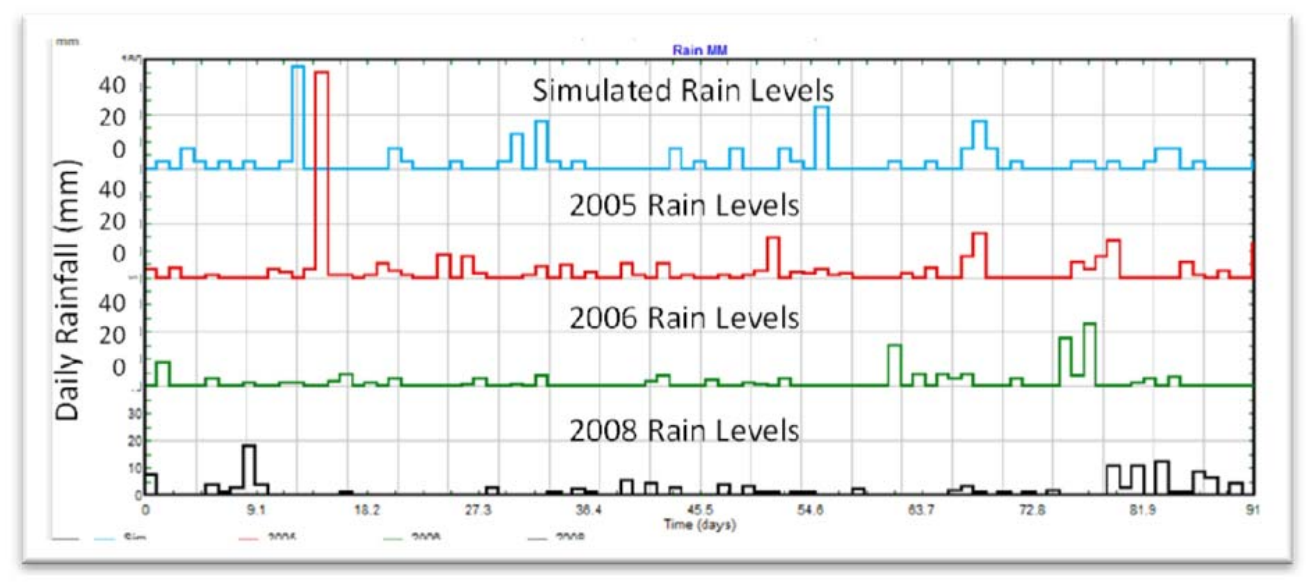

Figure 4: Andersen AFB Guam Actual Measured vs. Simulated Rain Levels (Spring)

\subsection{Rain Attenuation}

Two key dynamics for rain attenuation used in this paper are the slant range (or line of sight distance) from the satellite to the ground station/receiver and rain rate. Slant range is determined geometrically from the angle of elevation above the horizon. It is minimal for an elevation angle of 90 degrees (straight up) in which case the atmosphere through which the signal travels will be minimal $(\sim 130 \mathrm{Km})$. For lower elevation angles, the slant range between receiver and satellite will increase trigonometrically down to 0 degrees, although for all practical purposes, most communication will be ineffective for elevation angles of 5 degrees and less due to terrain obscuration and ever increasing attenuation effects. For this paper communication is considered to have no loss of data at and above 5 degree elevation angles unless there is rain attenuation.

As an example in Figure 5, consider the cases of elevation angles of 45, 30 and 5 degrees elevation angles. For an elevation angle (EA) of 45 degrees, the distance of atmosphere through which the signal must travel would be 1/0.707, or 1.414 that of the case for an elevation angle of 90 degrees (straight up). Therefore the signal travels through $41 \%$ more rain than for the 90 degree case. It gets worse for the 30 degree elevation angle case, where the slant range distance is now twice that of the vertical, and for the extreme case of a 5 degree elevation angle, in which case the signal would have to travel through approximately 11.5 times as much atmosphere.

The subject of satellite signal attenuation due to rain effects, better known as "rain fade", is a complex problem that negatively affects the ability to communicate between satellites and ground stations during extreme rain conditions (Hassan 2005, Roddy 2006, Wertz 2011). A proper understanding of these effects is necessary to understand the constraints on satellite communications with ground stations located in particularly tropical locations prone to large amounts of rainfall at unexpected times. For this paper Andersen AFB Guam is the example receptor with significant rain. The other two receptors at McMurdo Antarctica and Svalbard Norway are modeled assuming no data loss due to rain.

The susceptibility of a RF signal to rain fade is primarily dependent on four factors. These include the communication frequency band (e.g., $\mathrm{Ku} 12-18 \mathrm{GHz}$ ), the mean sea level height of the ground station, the intensity of the rainfall, and the elevation angle from the receiver to the satellite (Froehlich 1997). The band used will determine the types of attenuation effects that will be encountered due to signal scattering as the signal passes through a drop of rain. 


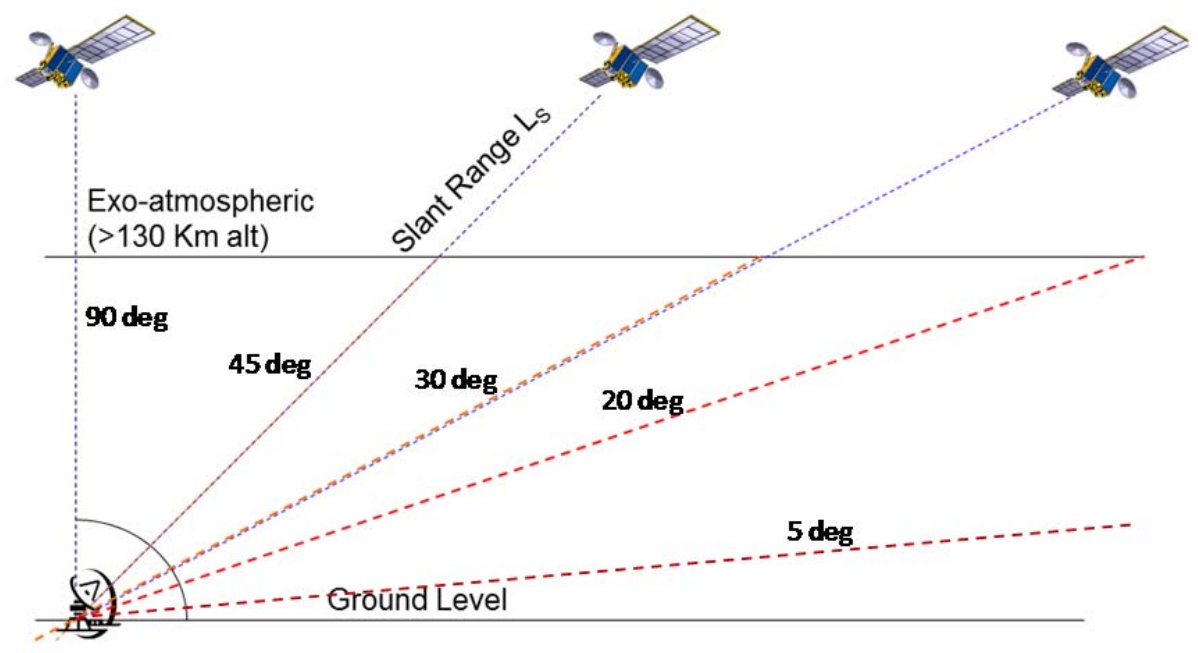

Figure 5: Satellite Slant Range

For example as seen in Figure 6, a C-band signal at an elevation angle of $5^{\circ}$ will be less susceptible to the effects of rain due to its wavelength of approximately $7 \mathrm{~cm}$, as compared to a typical $\mathrm{Ku}$ band frequency with a wavelength of about $2 \mathrm{~cm}$. A raindrop in the path of either signal that was close to half of the wavelength of the signal in diameter will cause signal attenuation. This is also why Ku band attenuation in rain is approximately nine times the attenuation for an equivalent $\mathrm{C}$-band transmission in the same conditions, or 9:1 dB where each $3 \mathrm{~dB}$ decrease represents a decrease by half in the power. This paper assumes a downlink frequency of $\mathrm{Ku}$ band at $14 \mathrm{GHz}$ with rain attenuation as a function of dynamic elevation angle and modeled rain rate.

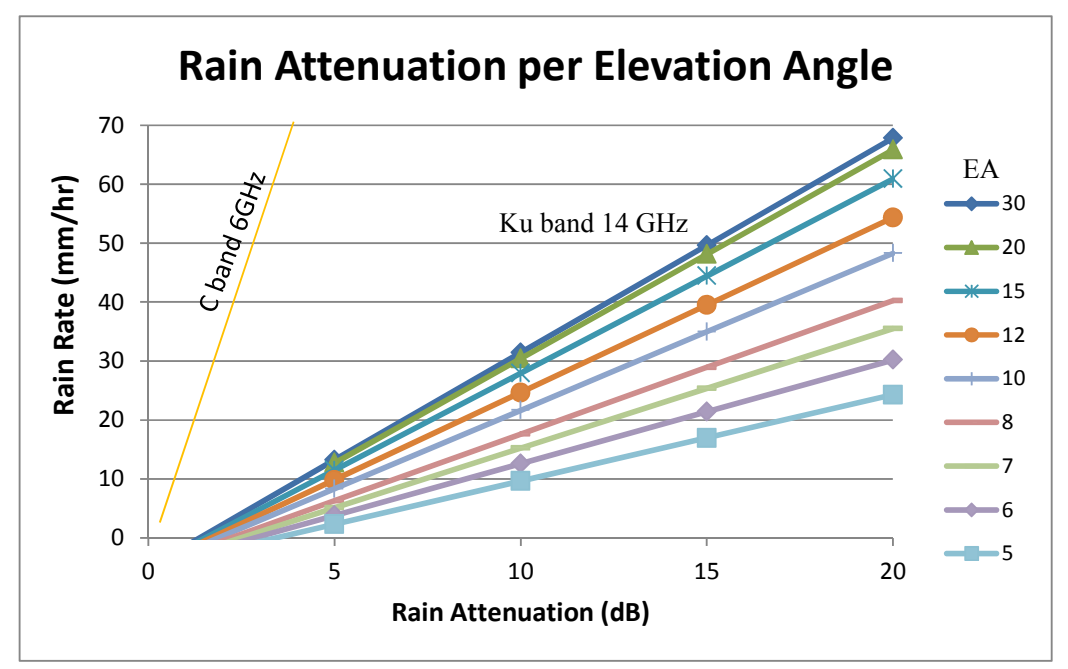

Figure 6: Rain Attenuation vs Rain Rate for $\mathrm{Ku}$ and $\mathrm{C}$ Bands

\subsection{Rain Attenuation Model}

Table 1 below was derived from the rain attenuation model described in section 2.2 for Ku band at 14 GHz. For a selected link margin $(\mathrm{dB})$ in Table 1, the simulation in section 3.2 interpolates the elevation angle (EA) for data loss using the dynamic rain rate $(\mathrm{mm} / \mathrm{hr}$ ) modeled in section 2.1. In clear weather the 


\section{Shannon and Marymee}

signal strength and link margin are sufficient to collect data at $5^{\circ}$ elevation angle without loss. The simulation uses this table with the simulated rain rate to derive the elevation angle at which and below which data is lost. Any data that the satellite transmits to the ground receptor at the start and end of contact, between $5^{\circ}$ to the calculated elevation angle, is marked "lost" by the simulator and must be recovered in a later contact. This recovery delays the weather data and in some cases after approximately three orbits, the data is permanently lost.

Table 1: Maximum Tolerable Rain Rates ( $\mathrm{mm} / \mathrm{hr}$ ) for Various Elevation Angles at Selected Link Margins $(\mathrm{dB})$

\begin{tabular}{|c|c|r|r|}
\cline { 2 - 4 } \multicolumn{1}{c|}{} & \multicolumn{3}{|c|}{ Link Margin (dB) } \\
\hline EA & 20 & 15 & 10 \\
\hline \hline 5 & 24.3 & 17.0 & 9.6 \\
\hline 6 & 30.2 & 21.4 & 12.6 \\
\hline 7 & 35.5 & 25.4 & 15.2 \\
\hline 8 & 40.3 & 28.9 & 17.6 \\
\hline 10 & 48.3 & 35.0 & 21.6 \\
\hline 12 & 54.3 & 39.5 & 24.7 \\
\hline 15 & 60.9 & 44.4 & 27.9 \\
\hline 18 & 64.5 & 47.1 & 29.8 \\
\hline 20 & 65.8 & 48.1 & 30.4 \\
\hline 25 & 66.9 & 48.9 & 30.9 \\
\hline 30 & 67.8 & 49.6 & 31.4 \\
\hline
\end{tabular}

\section{SIMULATED SENSOR DATA LOSS FROM RAIN ATTENUATION}

SatCom is a discrete event simulation of polar-orbiting weather satellites and sensor data retrieval built using ExtendSim from Imagine That Inc.

\subsection{Satellite Communication Simulation (SatCom)}

SatCom models the satellite sensor data collection and data retrieval supporting data latency analysis and architectural trades. In this paper SatCom models three weather satellites, stored sensor data on-board, and data downlinks to global receptors. The Satellite Tool Kit (STK) generated the orbital tracks and scheduled contacts for all satellites with the three ground receptors: Svalbard Norway, McMurdo Antarctica, and Andersen AFB Guam. The simulated satellite sensor data is collected continuously, stored in on-board recorders and transmitted downlink to the three receptors at all possible contacts. Data is buffered at the receptors, retrieved via land-based fiber networks and processed at CONUS ground facilities. SatCom models the orbital and global weather data that is collected by on-board sensors so that data retrieval and ground processing are complex and dynamic. SatCom models the data loss due to simulated rain at Guam and the added latencies for data recovery at the only recovery site in Svalbard, way. The lost data is recovered by command if there is sufficient time for command preparation in one of the next orbital passes over the normally clear Svalbard. This analysis is a notional comparison of data latency from the satellite's sensor collection to the data arrival at the processing facility and in this paper does not address the ground data processing of weather sensor data.

In Figure 7 SatCom models three notional weather satellites in polar orbits (inclination $98^{\circ}$ retrograde orbits) with contacts at Svalbard and McMurdo on each orbit and with less frequent contacts over Guam. The satellite on-board solid state recorder volume is shown in the bottom graph in Figure 7 with sensor collection volume in megabytes (MB) reflecting dynamic day/night sensor data rates. 


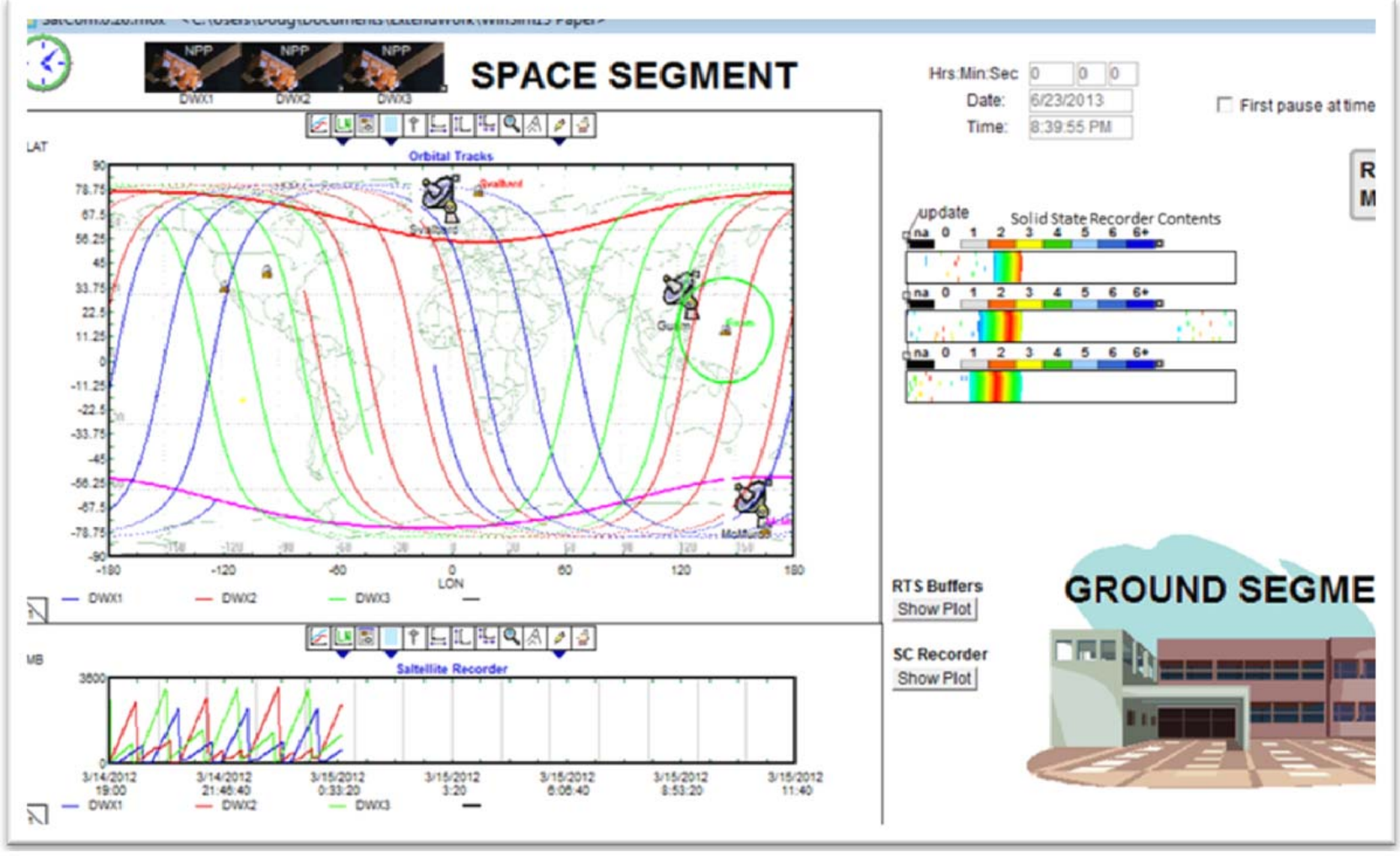

Figure 7: ExtendSim Model of Three Polar Orbiting Satellites and Satellite Recorder Volume (MB)

Figure 8 shows the sensor data that is recorded on-board the three satellites and stored until the next receptor contact. The color map shows the stored data over approximately one half of an orbit between Svalbard and McMurdo; the red packets were collected over the equator and blue over the poles. Any lost data is shown as single scattered packets. During the next contact with Svalbard, if that data is recovered, the color map clears these packets. If the data cannot be recovered, it will be overwritten by new sensor data in approximately three orbits.

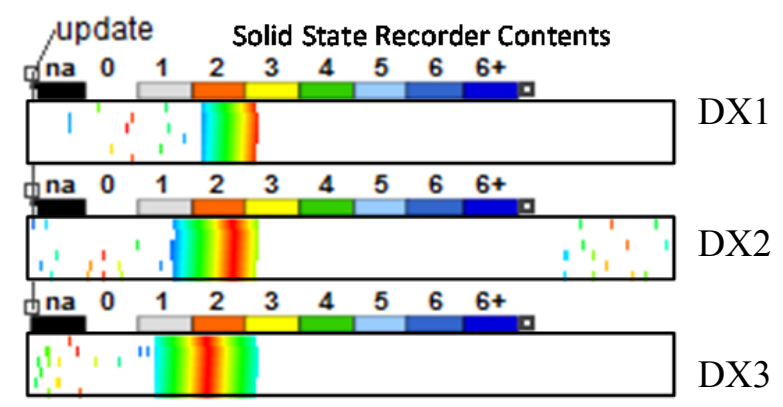

Figure 8: On-board Solid State Recorder Color Map Status and Content

Figure 9 shows the volume of data received at the three ground receptors. The top line (blue and using the right-side $\mathrm{Y}$ axis) shows the data received and buffered at Guam that is susceptible to rain attenuation. If transmitted data is lost but discovered in time (e.g., 30 mins) prior to the Svalbard contact, it can be retransmitted from the satellite recorder during that orbit. If there is insufficient time for command 


\section{Shannon and Marymee}

preparation, the recovery will require a second orbit and a longer latency for that data. Trade analyses in section 4 discuss the latency impact from data loss for alternative designs with and without Andersen AFB Guam.

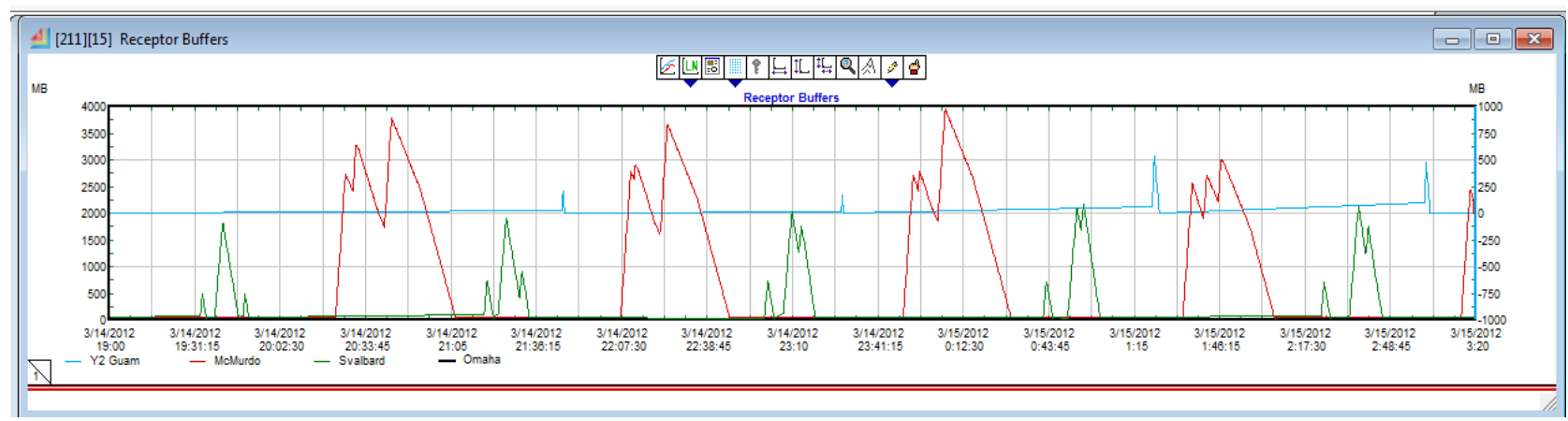

Figure 9: SatCom: Guam, McMurdo and Svalbard Ground Receptor Buffered Data (MB)

\subsection{Modeling Rain Attenuation, Data Loss and Data Recovery}

The rain attenuation model in Table 1 defines the elevation angle for data loss at specific rain rates. The simulation interpolates for the modeled rain rate (section 2.1) for the day and time of satellite contact. Sensor data in the downlink at and below the data-loss elevation angles are tagged as lost in the downlink data stream. The satellite is not aware of the data loss and continues to stream data during the satellitereceptor contact from start to end. The receptor model receives and buffers good data and in this model, discards corrupted, tagged-lost data.

SatCom retains "lost data" in satellite recorder model for a future contact at the system recovery and command receptor Svalbard; the data recovery model assumes no weather outages and data loss at Svalbard. SatCom models the satellite recorder volume and purges "lost" data when the recorder overwrites sensor data; this is an important consideration for data reliability. The recovered data is downlink and buffered along with recent sensor data and sent to the weather data processing facility. Latency for lost and recovered data adds significant aging to the average data latency. Alternate designs can compare processing recovered data first, as available, or last using different data processing priorities.

\section{ARCHITECTURAL TRADES AND ANALYSIS}

SatCom with the rain attenuation model simulates system performance, data latency and data reliability to consider and compare new receptor locations for autonomous downlinks. Performance analyses can compare the data latency improvement for different and added receptors versus the impact due to rain attenuation and data loss during autonomous downlinks. Data reliability analysis for autonomous downlinks requires modeling recovered data in subsequent orbits before the "lost" data is overwritten and permanently lost. There are numerous architectural alternatives for transmitting data blindly in autonomous downlinks. For example, what are the positive and negative impacts to data latency and reliability by raising the default start of contact elevation angle above 5 degrees during wet seasons. This tools supports the analyses for retrieving sensor data for ground processing in a timely, reliable way against the costs of overbuilding the ground architecture. 


\section{REFERENCES}

FreeMeteo.com - daily weather history for ANDERSEN AFB: http://www.freemeteo.com/default.asp?pid=155\&gid=9002379\&la=1\&sid=912180

Froehlich, Fritz E., and Kent, Allen, Encyclopedia of Telecommunications, Volume 14, New York, Marcel Dekker, Inc., 1997.

Hassan, Syed Idris Syed. Satellite Link Impairment Due to Rain and Other Climatic Factors. University of Malaysia, October 2005. http://www.aprsaf.org/data/aprsaf12_data/day2/csa/6_Dyed_csawg.pdf

Imagine That Inc., ExtendSim 7 User Guide, San Jose, CA. 2007.

Lander, Mark A., and Charles P. Guard, Creation of a 50-Year Rainfall Database, Annual Rainfall Climatology, and Annual Rainfall Distribution Map for Guam. University of Guam Water and Environmental Research Institute of the Western Pacific, Technical Report No. 102, June 2003. http://catalogue.nla.gov.au/Record/3640730

Roddy, Dennis. Satellite Communications, 4th ed. New York: McGraw-Hill, Inc., 2006.

Shannon, Doug. CPU Sizing vs. Latency Analysis: FTS EDR Latency Simulation, March 2008. http://www.extendsim.com/downloads/models/sols_npoess.ppt

Wertz, James R., David F. Everett, and Jeffrey J. Puschell, Space Mission Engineering: The New SMAD (Space Technology Library, Vol. 23), Hawthorne Microcosm Press, 2011.

\section{AUTHOR BIOGRAPHIES}

DOUGLAS C. SHANNON is senior modeling and simulation engineer with GreenDart, Inc. He developed performance models for weather satellite sensor data retrieval and ground data processing measuring data latencies, data reliability and sensor data ground processing capacity for the National Polar-orbiting Operational Environmental Satellite System (NPOESS) and Suomi National Polar-orbiting Partnership (NPP) programs. His email address is Douglas.Shannon@GreenDart.aero.

RICHARD K. MARYMEE is a lead systems engineer with GreenDart, Inc. specializing in space system and launch vehicle modeling and simulation, responsible for creating a system simulation of a proposed defense weather satellite system architecture. Richard was the interceptor flight kinematics lead engineer for most of his 15 years working on the Ground-based Midcourse Defense System, previously known as National Missile Defense system, with Boeing Defense Systems. Previous to that he supported the DoD working with program managers on quality engineering for naval weapons systems in Corona, CA. His email address is Richard.Marymee@GreenDart.aero. 